\title{
The Semon Club: the past and the future
}

\author{
E. B. Chevretton, M.S., F.R.C.S., A. G. Bibas, M.Sc., D.M., F.R.C.S.I. (Otol)
}

Sir Felix Semon (1849-1921) was born in Danzig, the eldest son of a German stockbroker. His medical studies at Heidelberg University were interrupted by the FrancoGerman war, when he served as a volunteer. He graduated in Berlin in 1873, settled in London a year later and joined the Hospital for Diseases of the Throat, Golden Square in 1877. He was appointed Physician to the Throat Department at St Thomas' Hospital in 1882 and in 1888 he became ENT Surgeon at the National Hospital, Queen Square. He made notable scientific contributions, that include his pioneering research on laryngeal paralysis (Semon's law) and laryngofissure for carcinoma of the larynx. He was the founder of the Laryngological Society of London and was elected its first President. So respected was Semon by his peers that he was made a Fellow of the Royal College of Physicians in 1885 and when he withdrew from active practice, the Semon lectures were established to perpetuate his name in 1913 , followed by the Semon Club meetings in $1958 .^{1-4}$

The Semon Club was started at the Royal National Throat Nose and Ear Hospital in 1958 to allow Otolaryngology Consultants and Registrars the opportunity to present and discuss unusual cases with their colleagues. Not surprisingly, the documentary evidence of the Semon Club meetings is incomplete. George Buchanan, Secretary from 1977 to 1998, has kindly put together the available minutes. It appears that Miles Foxen, who was the first secretary, was succeeded by Anthony Radcliffe and then by Bill Doey. Bill McKenzie took over in 1964, and for the first time careful minutes were kept from the 18th meeting in June 1964 to the 43rd meeting in November 1972. There appear to be no minutes prior to 1964 , and there is also a gap between November 1972 and May 1977.

Perusal of the minutes has revealed some fascinating anecdotes. In the 38th meeting (March 1971) Mr McKelvie presented a patient who had 'diphtheria at two years with numerous plastic repairs of the resultant tracheal and laryngeal stenosis. Skin was used and hair presents into the capacious lumen. A high pharyngeal stenosis persists; currently he has an indwelling portex tube, wired in place, proposed duration six months, which he resents. He has a temporary tracheostome. Members were interested; the Munchausen aspects of his condition were noted. Abbey had removed hair from the trachea in the past. All members noted that he might well come their way'. In the 39th meeting (June 1971) Mr Morrison presented a patient with a 'successful tympanoplasty. A testimony to the registrars. Good air bone gap closure was achieved, the hallmark of the case being division of a tympanosclerotic stapedius tendon. Shah indicated that this was commonplace. Taste changes were discussed, staging of tympanoplasties argued. McKenzie was missed at this point.' In the 40th meeting (November 1971) 'Pease presented a young man with highpitched voice. The examination of the larynx did not show any abnormality. Mares said his own voice was high pitched when he was young and he had speech therapy. Wallace had a patient - a young girl - who had just the opposite. Foxen suggested that he should have speech therapy. Citron felt he should also have benefit of a psychiatric consultation'.

Since 1998, the Semon Club has been hosted by Guy's Hospital by the attending Consultants. The plan is to run the meetings twice a year (May and October) on a Friday afternoon. The meetings are CME approved and the editors of the Journal of Laryngology and Otology have kindly agreed to publish the proceedings in abstract form. All colleagues are warmly welcomed.

\section{References}

1 McBride P. The Semon Lectures: Sir Felix Semon - his work and its influence on laryngology: Lecture 1.J Laryngol Otol 1913;28:113-29

2 McBride P. The Semon Lectures: Sir Felix Semon - his work and its influence on laryngology: Lecture II. $J$ Laryngol Otol 28: 169-87

3 Semon F. The Autobiography of Sir Felix Semon. London: Jarrolds Publishers, 1926

4 Harrison D. Felix Semon (1849-1921) A Victorian Laryngologist. London: The Royal Society Medicine Press Ltd., 2000

Proceedings of Semon Club 22/10/1999

Otolaryngology Department, Guy's Hospital

Chairman: Ms E. B. Chevretton, Consultant Otolaryngologist, Guy's \& St Thomas' Hospitals

Pathologist: Professor L. Michaels, University College London

Radiologist: Dr W. Jan, Clinical Fellow in Radiology, Guy's Hospital

Minutes: Mr A. Bibas, Specialist Registrar in Otolaryngology, Guy's \& St Thomas' Hospitals.

\section{Case 1: Progressive bilateral hearing loss and tinnitus following a head injury}

A 46-year-old patient was presented with a six-month history of progressive but intermittent bilateral hearing loss and tinnitus first noted after a head injury. Abnormal findings included an abnormal gait with eyes closed and an increase of the patient's tinnitus on performing Hallpike's manoeuvre to the right. A pure tone audiogram showed no recordable hearing on the left and a moderate degree of sensorineural hearing loss on the right of $50 \mathrm{~dB}$ across the frequencies with limitation of dynamic range in both ears. This was confirmed by BSER findings. Computed tomography (CT) scan showed exostosis above the right internal auditory meatus and magnetic resonance image (MRI) scan showed a cyst like lesion eroding the petrous bone below the right internal auditory meatus displacing the flocculus and IXth cranial nerve.

Discussion: It was suggested that otoacoustic emissions might be tried, although in the presence of sensorineural hearing loss they might be negative anyway.

Diagnosis: No definite diagnosis could be arrived at. Presented by Sava Soucek, St Mary's Hospital, London. 


\section{Case 2: Laryngeal sarcoidosis}

A 34-year-old female was presented with a three-month history of worsening cough, hoarseness and orthopnoea. Microlaryngoscopy revealed an oedematous supraglottis with sparing of the glottis; histology of the area showed florid chronic inflammation with numerous granulomas. Biochemical, immunological, microbiological and radiological investigations were all normal. The patient was diagnosed with laryngeal sarcoidosis in view of the histology and clinical pattern of the disease.

Discussion: A response to steroids was good although some chronic inflammatory changes in the larynx remain. A short video sequence of the changes in appearance of the larynx throughout the treatment was then shown. This was followed by a discussion on the different modalities which may be of help in diagnosing the condition, although no one test is diagnostic. The case was presented to show the difficulty in diagnosing single organ sarcoidosis.

Presented by Ann Hitchings, for H. Grant, Royal National Throat, Nose and Ear Hospital, London

\section{Case 3: Nasal mucormycosis}

A 32-year-old male was presented with a three-month history of right nasal congestion, one-month history of visual disturbances and proptosis, and hyposmia. CT scan revealed extensive soft tissue occupying the maxillary, frontal, sphenoidal, ethmoidal sinuses, and the orbit with destruction of the medial orbital wall and the cribriform plate. An endoscopic clearance was performed using a miocrodebrider. Microbiological and histological examination showed broad hyphae consistent with mucormycosis.

Discussion: The patient subsequently underwent two further endoscopic intranasal procedures for local recurrence. A recent repeat computed tomography (CT) scan showed that the disease is now under control. Professor Michaels held the opinion that the patient was most likely suffering from allergic fungal disease and that mucormycosis was simply a concomitant. This case illustrates that it is sometimes difficult to accurately diagnose and treat fungal diseases of the nose and paranasal sinuses.

Presented by Ricardo Persaud, for N. Salama, Lewisham University Hospital.

\section{Case 4: A rare case of an intranasal schwannoma in a young woman}

A 24-year-old female was presented with a one-year history of right-sided nasal obstruction, rhinorrhoea, anosmia, headache and recurrent minor epistaxis. Examination of the nose revealed a mucopurulent discharge and a right-sided polypoidal mass. The clinical findings were confirmed by a CT scan and the patient underwent a complete intranasal excision of the intranasal mass. Histology revealed the stroma to be composed of fairly uniform spindle cells arranged in loose stroma. Nuclei were arranged in a palisaded pattern (Verocay bodies). The appearance was consistent with a benign schwannoma
(Antoni type A). Positive immunoreactivity for S-100 protein confirmed the diagnosis.

Discussion: Twelve months post-operatively the patient was asymptomatic with no clinical evidence of recurrence.

Presented by Simon Gane, for E. Chevretton, Guy's and St Thomas' Hospitals, London

\section{Case 5: Wegener's granulomatosis and CSF leak}

A 59-year-old female was presented with a history of nasal obstruction, rhinorrhoea, epistaxis, and facial pain. cANCA was positive and a biopsy confirmed the diagnosis of Wegener's granulomatosis.

Discussion: The disease manifested itself by way of pulmonary cavitation, left main bronchus stenosis, nasal collapse, sinusitis and a unilateral conducive deafness. There was no renal involvement. Treatment was with pulsed cyclophosphamide initially and controlled by prednisolone and methotrexate. Unfortunately after four years there was a worsening of the sinonasal and respiratory symptoms, cyclophosphamide was recommenced. Recently facial pain and rhinorrhoea led to a suspicion of CSF leak, which was confirmed by beta 2 transferrin. A CT scan demonstrated a large defect in the skull base in the posterior ethmoid region. Plans for this to be repaired endoscopically have since been completed successfully.

Presented by Genevieve Rogers, for D. Roberts, Guy's Hospital, London

\section{Case 6: Multiple paragangliomata}

A 29-year-old hairdresser was presented with a two-year history of left pulsatile tinnitus. Otoscopy showed a mass behind the tympanic membrane suggesting a glomus tumour. All cranial nerves intact. A magnetic resonance image (MRI) scan of the skull base was performed and confirmed a mass in the middle ear. Carotid angiograms were performed, which showed bilateral vascular masses at the carotid bifurcations, suggesting carotid body tumours. Urinary analysis showed these tumours to be nonsecretory.

Discussion: It was necessary to preserve both vagus and hypoglossal on at least one side and as the right side had only one tumour the right carotid body tumour was initially excised. The post-operative recovery was good with no neurological deficit.

The patient later underwent excision of the left carotid body tumour and the glomus jugulare as a single procedure. Post-operative recovery was good with only a mild left accessory nerve palsy. Histology confirmed bilateral carotid body tumours and a left glomus tumour. On closer questioning, the patient admitted that her brother had had a carotid body tumour excised five years earlier.

Presented by Dia Samuel, for Professor M. Gleeson, Guy's Hospital, London 